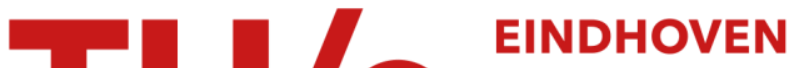 UNIVERSITY OF TECHNOLOGY
}

\section{Cepstrum analysis as a useful supplement to spectrum analysis for gear-box monitoring}

\section{Citation for published version (APA):}

Kraker, de, A., \& Stakenborg, M. J. L. (1986). Cepstrum analysis as a useful supplement to spectrum analysis for gear-box monitoring. In $\mathrm{H}$. Wieringa (Ed.), Experimental stress analysis : proceedings of the 8th international conference, Amsterdam, the Netherlands, May 12-16, 1986 (pp. 181-190). Nijhoff.

\section{Document status and date:}

Published: 01/01/1986

\section{Document Version:}

Publisher's PDF, also known as Version of Record (includes final page, issue and volume numbers)

\section{Please check the document version of this publication:}

- A submitted manuscript is the version of the article upon submission and before peer-review. There can be important differences between the submitted version and the official published version of record. People interested in the research are advised to contact the author for the final version of the publication, or visit the $\mathrm{DOI}$ to the publisher's website.

- The final author version and the galley proof are versions of the publication after peer review.

- The final published version features the final layout of the paper including the volume, issue and page numbers.

Link to publication

\section{General rights}

Copyright and moral rights for the publications made accessible in the public portal are retained by the authors and/or other copyright owners and it is a condition of accessing publications that users recognise and abide by the legal requirements associated with these rights.

- Users may download and print one copy of any publication from the public portal for the purpose of private study or research.

- You may not further distribute the material or use it for any profit-making activity or commercial gain

- You may freely distribute the URL identifying the publication in the public portal.

If the publication is distributed under the terms of Article 25fa of the Dutch Copyright Act, indicated by the "Taverne" license above, please follow below link for the End User Agreement:

www.tue.nl/taverne

Take down policy

If you believe that this document breaches copyright please contact us at:

openaccess@tue.nl

providing details and we will investigate your claim. 

CEPSTRUM ANALYSIS AS A USEFUL SUPPLEMENT TO SPECTRUM ANALYSIS FOR GEAR-BOX
MONITORING

\author{
A. DE KRARER AND M.J.I. STAKENBORG
}

EINDHOVEN UNIVERSITY OF TECHNOLOGY, THE NETHERLANDS

\title{
SUMMARY
}

Cepstrum analysis is used frequently in data processing and signal analysis. The use of this new technique however in monitoring the mechanical condition of gearboxes is still rather unknown. This paper presents the results of an evaluation of the cepstrum technique as a tool for the interpretation of gearbox spectra. The origin of vibrations in gearboxes and a useful definition of the cepstrum will be presented. The method will be applied for the condition evaluation of a transmission train of 4 gears and it will be shown that the method can be used effectively if some practical considerations and some limitations are kept in mind.

\section{INTRODUCTION}

In general, most (rotating) machinery such as gearboxes generate vibrations and the corresponding vibration spectra show a characteristic form when the mathine is in good mechanical condition. Changes in these vibration spectra are often an indication that the condition of the machine is changing for example due to wear or a toothdamage. A well known and very often used method to evaluate the mechanical condition is normal spectral analysis by means of measuring the vibration of a machine with the help of accelerometers on the housing. This condition monitoring is based on the assumption that, providing the operating conditions have not been changed, any change in vibration behaviour could be an indication of an impending failure. Although this assumption will not always be correct, the condition monitoring has proven to be of great use for the monitoring of e.g. turbine blades, bearings and gearboxes. In 1963 Bogert [1] introduced a new technique in signal analysis called the Cepstrum Analysis as a numerical operation working on measured spectra. This technique is now used frequently in different areas of data processing and signal analysis but the use in gearbox monitoring has left many questions. Some of these questions are dealing with the typical characteristics of gearbox vibration spectra and the origin of vibrations, practical considerations of using the cepstrum technique and the advantage of using the cepstrum in evaluating the changes in vibration spectra of a typical gearbox and the relation of these changes with a possible decreasing condition.

\section{VIBRATIONS IN GEARBOXES}

The study of the vibrational behaviour of gearboxes has received moderate attention in literature but due to the great complexity of gear dynamics, it remains a poorly understood area. In practice not only the usual out of balance and bearing forces are active in gear dynamics but also the precise geometry of the gear profiles has a crucial effect on the vibrational behaviour. In general flexural vibrations will be more important than torsional 
vibrations because flexural vibrations are transferred directly to the housing via the bearings. Also coupling between flexural and torsional vibrations is usual and should be taken into account under certain circumstances. with increasing frequency the nature of the lubricating oil film and the teeth flexibility become increasingly important. Looking at gearbox vibration mechanisms three of the most important vibration sources are:

- Time variations in the mess stiffness, caused by variation of the number of teeth in contact and variation in the stiffness of the individual teeth.

- Dynamical effects due to deviations from the ideal tooth profile. In practice all gears contain teeth manufacturing errors, such as errors due to the gear cutting process, deviations in the mesh angle, deviations from the involute profile, surface roughness of the gears etc.

- Oscillations in the sliding velocity. During the transmission of power there will be rolling and slipping in the point of contact and also oscillations may occur because of stick-slip effects.

Due to these mechanisms, an amplitude or frequency modulation of the vibration signal may be caused, resulting in sideband-structures in the spectrum around the toothmeshing frequency and its harmonics. This is illustrated in figure 1.

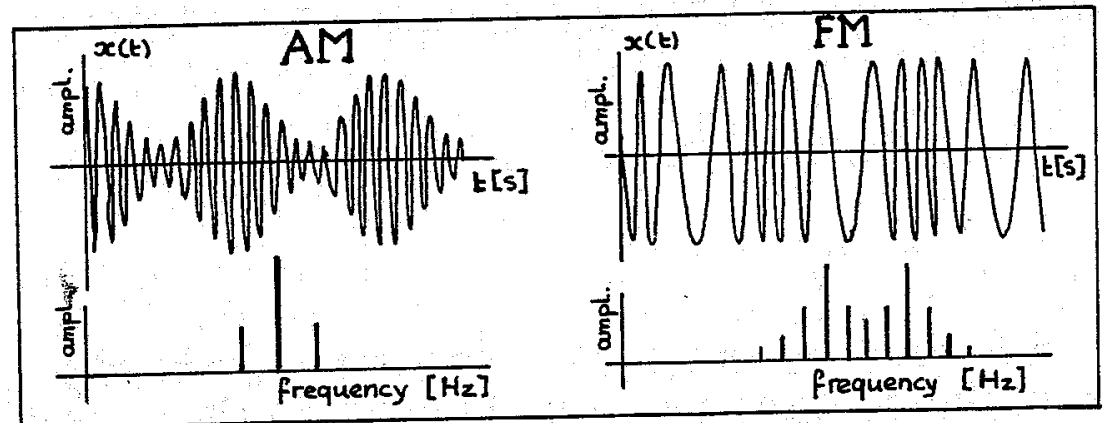

Figure 1. Amplitude- and Frequency modulated signals and their spectra.

Since in gears, not separable combinations of amplitude- and frequency modulation will lead to complex sidebandstructures in measured spectra a cepstrum analysis can offer a tool to improve the interpretation of such a spectrum.

\section{CEPSTRUM FUNDAMENTALS}

The cepstrum was first described by Bogert et al. [1] in 1963 as a technique for finding echo arrival times in a composite seismic signal. These authors defined the (power)cepstrum $C(T)$ as "the powerspectrum of the logarithm of the powerspectrum of a random proces", so:

$$
C(\tau)=\left|\int_{-\infty}^{\infty} \log s_{x x}(f) e^{-2 \pi j f t} d f\right|^{2}
$$

where $S_{x X}(f)$ is the two-sided auto powerspectrum of the random proces $x(t)$ :

$$
S_{X X}(f)=\lim _{T \rightarrow \infty} \frac{1}{T} E\left[X^{*}(T, f) X(T, f)\right]
$$


and $X(T, f)$ represents the finite Fourier Transform of $x(t)$ :

$$
X(T, f)=\int_{0}^{T} x(t) e^{-2 \pi j f t} d t
$$

For some reasons a number of slightly different definitions for the cepstrum are known in literature, such as

$$
\text { Amplitude Cepstrum } c(\tau)=1 \int_{-\infty}^{\infty} \log s_{x x}(f) e^{-2 \pi j f \tau} d f \mid
$$

or

$$
\text { Amplitude Cepstrum } C(\tau)=\left|\int_{-\infty}^{\infty} \log S_{x x}(f) e^{2 \pi j f \tau} d f\right|
$$

or

$$
\text { Complex Cepstrum } C(\tau)=\int_{-\infty}^{\infty} \log X(T, f) e^{2 \pi j f \tau} d f
$$

An important reason for using definition (5) instead of (1) is the relationship between the Cepstrum definition and the autocorrelation function $\mathrm{R}_{\mathrm{Xx}}(\tau)$, defined as:

$$
R_{x x}(\tau)=\int_{-\infty}^{\infty} S_{x x}(f) e^{2 \pi j f \tau} d f
$$

Comparing this expression with definition (5) we can note that the only difference is that in case of the cepstrum the logarithm of the spectrum values is taken before performing the inverse Fourier Transform. $\left(F^{-1}\right)$.

The powerspectrum $S_{x x}$ (f) of a vibration signal on a gearbox-housing is the product of the powerspectrum $S_{Y Y}(f)$ of the source function and the squared amplitude of the transfer function $\mathrm{H}_{\mathrm{yx}}(\mathrm{f})$ of the transmission path, i.e.: gears, shafts, housing, etc. So:

$$
S_{X x}(f)=S_{y y}(f)\left|H_{y x}(f)\right|^{2}
$$

Taking the complex cepstrum of $s_{x x}$ we get:

$$
C_{x}(\tau)=C_{y}(\tau)+C_{H}(\tau)
$$

where $C_{x}(\tau)$ respectively $C_{y}(\tau)$ are the complex cepstra of $x(t)$ respectively $y(t)$ and $C_{H}(\tau)$ is defined as: $C_{H}(\tau)=2 f^{-1}\left\{\log \left|H_{y x}\right|\right\}$. $E_{q n}$ (9) shows that the source and transmission path effects are additive in the cepstrum. In general, the amplitude of a transferfunction will have a different cepstrum content (different periodic behaviour) than the cepstrum image of the source function. It will therefore be possible to separate these two influences in the final cepstrum $C_{x}(\tau)$. 
To avoid confusion between spectrum- and cepstrum methodology Bogert et al, introduced the following paraphrased terms,

$\begin{array}{lll}\text { frequency } & \ldots & \text { quefrency } \\ \text { spectrum } & \ldots & \text { cepstrum } \\ \text { phase } & \ldots & \text { saphe } \\ \text { magnitude } & \ldots & \text { gamnitude } \\ \text { filtering } & \ldots & \text { liftering } \\ \text { harmonic } & \ldots & \text { rahmonic } \\ \text { period } & \ldots & \text { repiod }\end{array}$

Today, the most utilized terms are cepstrum, quefrency and rahmonic; e.g. magnitude in the cepstrum domain is often called magnitude and not gamnitude. The independent variable of the cepstrum $(\tau)$ has the dimension of time and it is called quefrency. This quefrency tells us something about frequency spacings in the spectrum.

4. A NEW CEPSTRUM DEFINITION

Although the cepstrum definitions according eqn (1), (5), and (6) are very useful in analysing speech or seismic signals, the analysis of gearboxes demands a slightly different definition of the cepstrum. To illustrate this need we consider the harmonic function $x(t)=A \cos \left(2 \pi f_{0} t+\varphi\right)$. The autopowerspectrum $s_{x x}(f)$ of this function will be:

$$
S_{x x}(f)=\frac{1}{4} A^{2}\left[\delta\left(f-f_{0}\right)+\delta\left(f+f_{0}\right)\right]
$$

According to the cepstrum definition of eqn (5) we get

$$
C_{X}(\tau)=F^{-1}\left\{\log S_{x x}(f)\right\}=\log \left(\frac{A^{2}}{2}\right) \cos \left(2 \pi f_{0} t\right)
$$

The functions $x(t), s_{x x}(f)$ and $c_{x}(r)$ are shown in fig 2 .
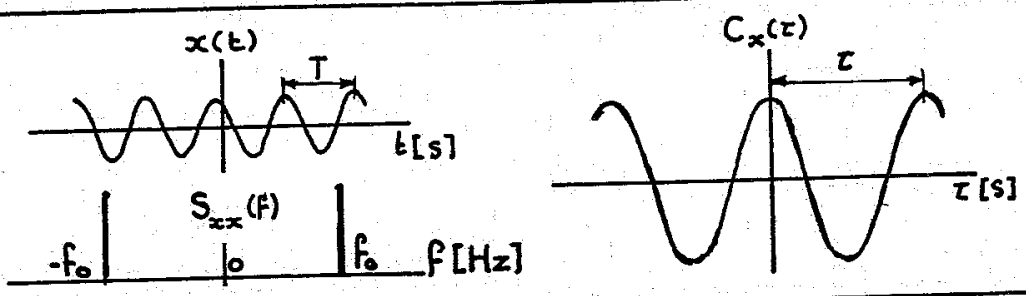

Figure 2. Time signal, spectrum and cepstrum of $x(t)=A \cos \left(2 \pi f_{0} t+\varphi\right)$.

The nonlinear amplitude filtering by taking the log-values of the powerspectrum is done to de-accentuate the larger amplitude frequency components (e.q. carrier frequencies) in order to give some more accent to lower amplitude components (such as modulated sidebands). So one could say that the powerspectrum is flattened, or whitened. Another advantage of the log operation is the separation of source function and transmission path effect as shown earlier. 
In analysing speech or seismic signals the amplitude peaks of interest in a powerspectrum will often occur at multiples of some frequency interval, so at frequencies $\mathrm{f}=\mathrm{k} \Delta[\mathrm{Hz}], \mathrm{k}=\ldots,-2,-1,0,1,2, \ldots$ In the corresponding cepstrum a distinct peak is then found at $\tau=1 / \Delta[s]$, as shown in fig 3 .

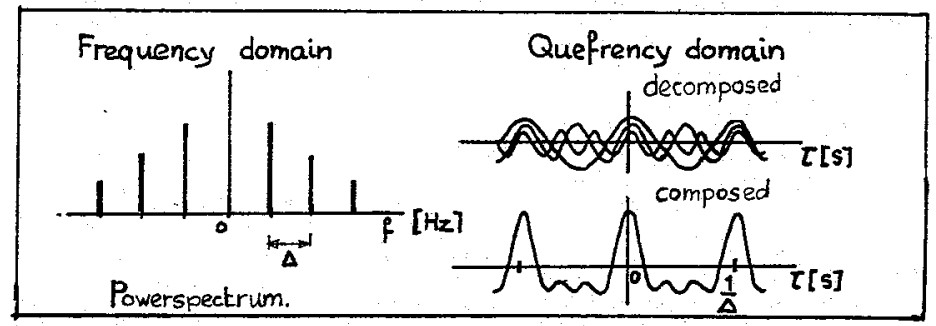

Figure 3. Hypothetical spectrum and its cepstrum.

In the powerspectra of gearbox vibrations a family of sidebands not necessarily go through zero frequency, in other words the first peak of a family of sidebands in the powerspectrum does not need to occur at $f=k \Delta[\mathrm{Hz}]$, as shown in fig. 4.

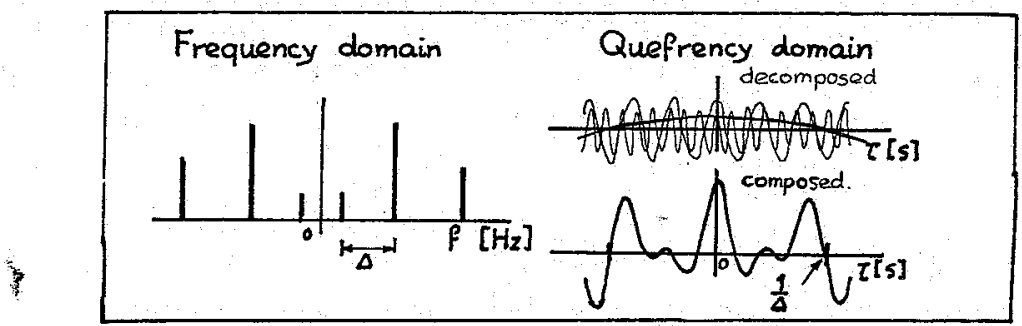

Figure 4. Hypothetical spectrum and its cepstrum.

In the cepstrum of $\mathrm{fig} .4$ a zero passing occurs at $i=1 / \Delta$ instead of a peak as in fig. 3. In relatively complex cepstra these zero passings indicating periodicities in the powerspectrum might be easily overlooked, especially when using absolute values as described in the cepstrum definitions (1) and (5). Another problem occurs when there are also other zero-passings present in the cepstrum which contain no information on sideband spacings as given in fig. 4. This indicates the need for an adaptation of the cepstrum definition when used for analysing gearbox vibration signals. In the formulation of this definition we need the Hilbert transform of which a short explanation will be given first.

A causal function $g(t) \quad(g(t)=0$ for $t(0))$ can always be decomposed into an even function $e(t)$ and an odd function $o(t)$. We then can write:

$$
\begin{aligned}
& e(t)=\operatorname{sgn}(t) o(t) \\
& o(t)=\operatorname{sgn}(t) e(t)
\end{aligned} \quad \operatorname{sgn}(t)=\left\{\begin{array}{rl}
1 & t \geq 0 \\
-1 & t<0
\end{array}\right.
$$

Thus in the time domain there exists a definite relationship between the even and odd part of a causal function. In the frequency domain there is also a relation between the real and imaginary part of the Fouriertransform $G(f)$ of the causal function $g(t)$. This relation is called the Hilbert transform. Let the Fouriertransforms of $g(t), e(t)$ and $o(t)$ be $G(f), R(f)$ and $j X(f),(R$ and $X$ real), then: 


$$
\begin{array}{ll}
G(f)=R(f)+j X(f) & \\
X(f)=-\frac{1}{\pi f} \otimes R(f) & \text { Forward Hilbert Transform } \\
R(f)=\frac{1}{\pi f} \otimes(f) & \text { Backward Hilbert Transform }
\end{array}
$$

where $\otimes$ is the convolution symbol.

We now return to the basic problem: the definition of the cepstrum and the formulation of an efficient way to calculate this cepstrum numerically. The aim was to detect periodicities in the (log-)powerspectrum $5_{x x}(f)$, being a real even function. To meet our wishes, we will use the cepstrum definition:

$$
c(\tau)=\mid F\left\{\left.\log s_{x x, 1}(f)\right|^{2}\right.
$$

with $S_{x x, 1}$ being the 1 -sided power spectrum:

$$
\begin{array}{ll}
s_{x x, 1}(f)=2 s_{x x}(f) & \text { for } f>0 \\
s_{x x, 1}(f)=s_{x x}(f) & \text { for } f=0 \\
s_{x x, 1}(f)=0 & \text { for } f<0
\end{array}
$$

This definition is based on three hypotheses (for a prove see [3]):

1) Calculating the cepstrum, the same result is obtained by a forward or inverse transform of the (log-) powerspectrum.

2) It is sufficient to transform the 1-sided spectrum only i.e. the positive frequency values of the (log-) powerspectrum, multiplied by a factor of 2 .

3) In some situations, the imaginary part of the cepstrum of a 1-sided powerspectrum, being the Hilbert transform of the real part, can be of great use.

The first two hypotheses are dealing with the use of standard signal analysis equipment for cepstrum analysis, reduction of computertime or the increase of the resolution in the calculated cepstrum by adding zero's to the 1 -sided spectrum. The third hypothesis makes it possible to avoid that zeropassings as characteristic points in the cepstrum might be overlooked because zero passings in the real-part of the cepstrum will correspond with peaks in the imaginary-part of the cepstrum.

\section{PRACTICAL CONSIDERATIONS USING THE CEPSTRUM ANALYSIS}

The cepstrum technique often demands some additional effort and consideration before it can be used succesfully in practice. In the following some practical considerations when using the cepstrum technique for the analysis of the vibration spectrum of gearboxes will be presented. When we start with a spectrum with $\mathrm{N}$ spectral lines then a standard FFT algorithm will result in a cepstrum with $\mathrm{N} / 2$ significant (complex) values. The resolution in the cord before performing the cepstrum computation. A DC-component in the trum (resulting for the cepstrum in a value $c(r=0)$ ) has no practical relvance which means that the mean value of the log spectrum can be set to zero before the calculation of the cepstrum. This is likely to reduce the jump (discontinuity) between the last spectrum line and the first appended zero 
and consequently also will reduce signal leakage. In order to reduce this signal leakage further one might be motivated to use a window-function to the log-spectrum before applying the FFT. Easily can be seen that Hanningtype windows will strongly affect the calculated cepstrum, especially when sideband structures of interest are in the beginning and/or end region of the spectrum. The window should therefore be relatively flat over that portion of the $\log$-spectrum containing periodicities. A cosine taper data window after Bingham [3] showed a satisfying behaviour.

In practice there will always be a base noise level in each spectrum which will have a direct effect on the amplitudes of the cepstrum components as indicated in fig. 5 .
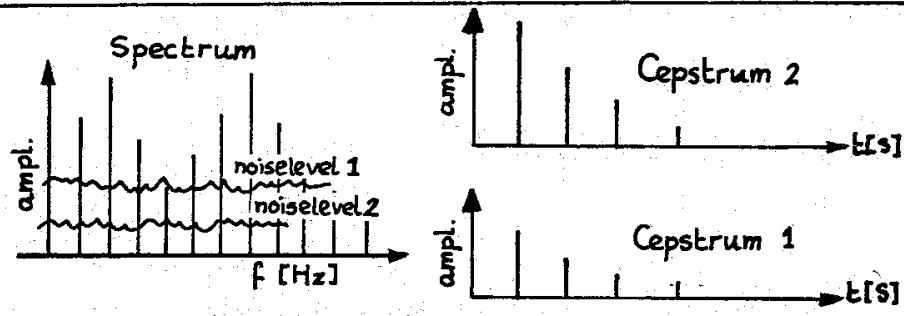

Figure 5. Influence of noise level on cepstrum results.

The consequence of this is that it is only valid to compare cepstra quantitatively if the base noise conditions are similar. Bridging occurs when two or more close frequency components in the spectrum are so close that their amplitude peaks cannot be separated and form one broad peak, in other words they aremelted together. The possibility of the cepstrum to separate sideband strưctures with only slightly different sideband spacings consequently will be affected. To be able to detect the smallest modulation frequency $f_{m}$ in a baseband spectrum the number of spectral lines $\mathrm{N}_{1 \text { ines }}$ for cepstrum calculation has to be

$$
N_{\text {lines }} \geq \frac{\mathrm{f}_{0} 2 N_{\text {harm }}}{f_{\mathrm{Im}}}
$$

where: $f_{0}=$ shaft rotational speed (often $f_{0}=f_{m}$ )

$\mathrm{Z}$ = number of teeth

$\mathbb{N}_{\text {harm }}=$ number of toothmesh harmonics in the spectrum

If for example, $z=40, N_{\text {harm }}=2.5, f_{0}=f_{m}$ we get $N_{1 \text { ines }} \geq 800$. It will therefore often be necessary to perform a zoom-spectrum analysis in order to obtain sufficient resolution in the original spectrum, before performing the cepstrum analysis. Some analysers have therefore the capability to perform a cepstrumanalysis of a spectrum of up to 4000 lines using a special method of zoom [5].

\section{PRACTICAL APPLICATION}

A special software program was developed, based on a Nicolet 100A FFT spectrum analyser in combination with a HP9826 desktop computer to perform a cepstrum evaluation. The program uses 4 neighbouring zoomspectra of 400 lines each to get a baseband spectrum with enough resolution. Another 448 
zero's were added after which a FFT transform on the 2048 spectral data was carried out, leading to a 1024 amplitude cepstrum according to eqn. (14).

To evaluate the use of the cepstrum technique a gearbox with a transmission train of 4 gears (see fig. 6) and a load of respectively $1.12,3.32$ and 5.48 $\mathrm{KW}$ was analysed.

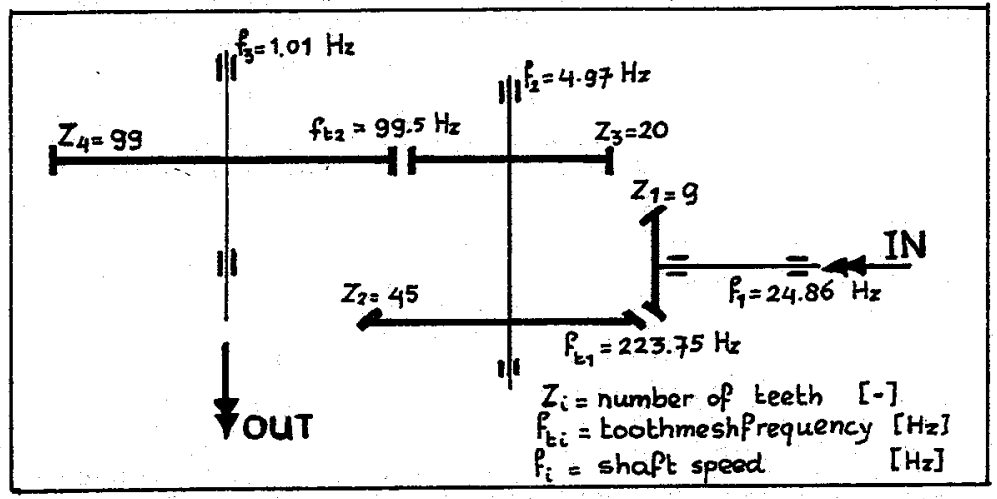

Figure 6. Picture of the gearbox.

Fig. 7a shows a vibrationspectrum at $1.12 \mathrm{KW}$, measured on the housing of one of the bearings. High vibration amplitudes can be found at the toothmesh frequency $f_{t 1}=223.75[\mathrm{~Hz}]$ and a smaller value at the toothmesh frequency $f_{t 2}=99.50[\mathrm{~Hz}]$. The corresponding cepstrum is shown in Fig. 7b. The abcissa gives the quefrency [sec] and also the reciproke value of it in [Hz].

a.

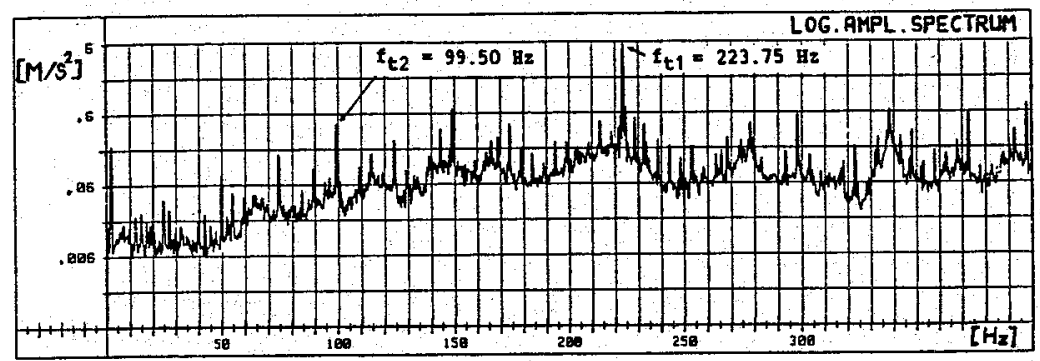

b.

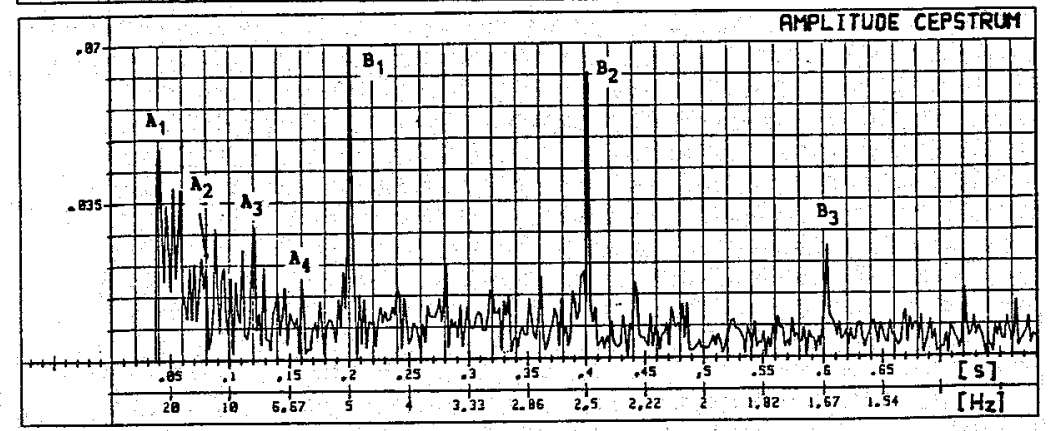

Figure 7. Measured spectrum and calculated cepstrum.

In the cepstrum a high amplitude $B_{1}$ at $\tau=0.201$ [sec] can be detected which 
indicates a strong modulation with $4.97[\mathrm{~Hz}]$, which is the shaft rotational speed of shaft 2. The peaks $B_{2}$ and $B_{3}$ are rahmonics of $B_{1}$. The peak $A_{1}$ at $\tau$ $=0.04$ [sec] indicates also a modulation with the shaft rotational speed of shaft $1(24.86 \mathrm{~Hz})$.

The peaks $A_{2}, A_{3}$ and $A_{4}$ are again the rahmonics of peak $A_{1}$. The relatively large amplitude of cepstrumcomponent $B_{1}$ argues the most serious modulation, namely with frequency $f_{2}$ of shaft 2 . The real fysical cause for this modulation cannot be located with this cepstrum technique. The observed phenomena however are an indication for:

- An unfavourable toothmeshaction at toothmeshfrequency $f_{t 1}$.

- Mounting errors or excentricity of the shaft with speed $f_{2}$.

After opening and inspection of the gearbox it turned out that the pignon wheel 2 was not mounted correctly on its shaft (schrink-fit).

The mounting error resulted in a periodic interference of the pitch circles with a frequency of 4.97 [Hz] leading to this clear amplitude and frequency modulation of the vibration signal. The practical consequence of this condition was a strong wear of the teethprofile which could be observed at the inspection of the gearbox after finishing the measurements.

\section{EVALUATION AND CONCLUSIONS}

Apart from the fact that the use of spectrum-cepstrum analysis techniques demand a considerable skill of the user to prevent misinterpretation some complications ofter occur when using this technique on gearboxes in practice:

- The spectra are very sensitive to small load changes and to changes in the rotational frequency,

- There will not always be a definite relationship between changes in the spectrum and the size of a possible mechanical defect,

- It is not always possible to obtain stationary signals for example in case of torque fluctuations, time varying transmission paths as found in epicyclic gearboxes.

- A bad signal to noise ratio complicates the interpretation of the spectra,

- Generalization and standardization is almost impossible. Each gearbox has to be analysed individually.

- It is only possible to compare cepstra qualitatively and not quantitatively.

- The presence of more than one series of rahmonics in the cepstrum might be confusing especially when they are very close.

Due to these complications the enthusiasm on the perspectives of the cepstrum analysis in gearbox monitoring should be somewhat tempered by a healthy scepticism. Nevertheless in many cases the cepstrum will be a useful supplement to the normal spectrum analysis for the detection and evaluation of sidebands in gearbox vibration spectra due to modulation. In order to make these technique more valid, research should be done to get more insight in the effects of mechanical defects or faults on the vibrational behaviour. This points out the need for adequate dynamical models for gearbox systems.

\section{Acknowledgement}

The autors wish to thank Ir. Bouwman for the support and the facilities provided by KEMA, Arnhem and $\mathrm{Ir}$. Toersen for his advise and ideas concerning gearbox monitoring and maintenance. 


\section{References}

[1] Bogert, B.P., Healy, M.J.R., Tukey, J.W., "The Quefrency Analysis of Time Series for Echoes: Cepstrum, Pseudo auto covariance, Cross Cepstrum and Saphe Cracking" " Proceedings of a Symposium on Time Series Analysis by M. Rosenblatt, (Ed.), Wiley, N.Y., 1963, pp. 209-243.

[2] Childers, D.G., Skinner, D.P., Remerait, R.C., "The Cepstrum: A Guide to processing", Proceedings of the IEEE, Vol. 65, no. 10, oct. 1977.

[3] Stakenborg, M.J.I., "On the Use of Cepstrum Analysis in Gearbox Monitoring", Report WFW 84.025, University Eindhoven, aug. 1984.

[4] Randall, R.B., "A new Method of Modelling Gear Faults", Inl. of Mech. Design, april 1982, Vol. 104/259.

[5] Thrane, N., "Zoom FFT", B\&R, Technical Review, no. 2, 1980. 